\title{
Temperature scaling in a dense vibro-fluidised granular material
}

\author{
P Sunthar and V Kumaran \\ Department of Chemical Engineering, \\ Indian Institute of Science, Bangalore, 560 012, India.
}

\begin{abstract}
The leading order "temperature" of a dense two dimensional granular material fluidised by external vibrations is determined. The grain interactions are characterised by inelastic collisions, but the coefficient of restitution is considered to be close to 1 , so that the dissipation of energy during a collision is small compared to the average energy of a particle. An asymptotic solution is obtained where the particles are considered to be elastic in the leading approximation. The velocity distribution is a Maxwell-Boltzmann distribution in the leading approximation. The density profile is determined by solving the momentum balance equation in the vertical direction, where the relation between the pressure and density is provided by the virial equation of state. The temperature is determined by relating the source of energy due to the vibrating surface and the energy dissipation due to inelastic collisions. The predictions of the present analysis show good agreement with simulation results at higher densities where theories for a dilute vibrated granular material, with the pressure-density relation provided by the ideal gas law, are in error.
\end{abstract}

\section{INTRODUCTION}

Recent developments in the physics of granular matter [1] have illustrated that the dissipative nature of the interactions between grains can result in a variety of different phenomena. Of particular interest in recent years has been the dynamics of vibrated granular materials [2, 3], which exhibit stationary states as well as waves and complex patterns. In order to describe these diverse states of the material, it is necessary to derive macroscopic descriptions by averaging over the microscopic details of the motion and interactions between individual grains. This goal has proved elusive, however, because a vibrated granular material is a driven dissipative system, and the interactions between the particles are characterised by a loss of energy due to inelastic collisions. The statistical mechanics framework developed for equilibrium or near equilibrium systems cannot be used in this case. Consequently, phenomenological models [6] have been used to describe the dynamics of granular materials. The kinetic theories developed for granular flows [7, 8, usually assume that the system is close to "equilibrium" and the velocity distribution function is close to the Maxwell-Boltzmann distribution.

Experimental studies and computer simulations have reported the presence of a uniformly fluidised state in a vibrated bed of granular material. Luding, Herrmann and Blumen [9] carried out 'Event Driven' (ED) simulations of a two dimensional system of inelastic disks in a gravitational field vibrated from below, and obtained scaling laws for the density variations in the bed. An experimental study of a vibrated fluidised bed was carried out by Warr, Huntley and Jacques [2]. Their experimental set up consisted of steel spheres confined be- tween two glass plates that are separated by a distance slightly larger than the diameter of the spheres. The particles were fluidised by a vibrating surface at the bottom of the bed, and the statistics of the velocity distribution of the particles were obtained using visualisation techniques. Profiles for the density and the mean square velocity were obtained, and the particle velocity distributions were also determined at certain positions in the bed. Both of these studies reported that there is an exponential dependence of the density on the height near the top of the bed, similar to the Boltzmann distribution for the density of a gas in a gravitational field. However, the dependence of the density deviates from the exponential behaviour near the bottom. The dependence of the mean square velocity on the vibration frequency and amplitude were found to be different in the two studies.

A theoretical calculation of the distribution function in a vibro-fluidised bed was carried out by Kumaran [8,10]. The limit of low dissipation, where the coefficient of restitution $e$ is close to 1 was considered. In this limit, the mean square velocity of the particles is large compared to the mean square of the velocity of the vibrating surface, and the dissipation of energy during a binary collision is small compared to the energy of a particle. A perturbation approximation is used, where the energy dissipation is neglected in the leading order approximation, and the system resembles a gas at equilibrium in a gravitational field. The velocity distribution function is a Maxwell-Boltzmann distribution, and the density decreases exponentially from the vibrating surface. The first order correction to the distribution due to dissipative effects was calculated using the moment expansion method, and the results were found to be in qualitative agreement with the experiments of Warr et. al. [2].

The theoretical predictions [8, 10] were compared with previous experimental and simulation studies by McNamara and Luding [11]. They found that the theory was in good agreement with experiments for dilute beds, where the area fraction of the particles is low, but there were systematic deviations from the theoretical predictions as the area fraction increases. This is to be expected, since the analysis assumed that the density is small and the pair distribution function was set equal to 1 and therefore the pressure is related to the density by the ideal gas law. These assumptions become inaccurate as the area fraction of the bed increases. An approximate method for including the correction to the pair distribution function was suggested by Huntley [12].

In the present analysis, the correction to the low density theory of Kumaran [8, 10] is determined for a vibro-fluidised bed where the coefficient of restitution is close to 1 . An asymptotic analysis is used, where the dissipation is neglected in the leading approximation. The leading order density and veloc- 
ity profiles are determined using the momentum balance equation in the vertical direction. In contrast to the earlier theory [8, 10], the virial equation of state for a non-ideal two dimensional gas is used to determine the leading order density profile. The density profile differs from the Boltzmann distribution, but the velocity distribution function is still a MaxwellBoltzmann distribution. The leading order temperature is determined by a balance between the source and dissipation of energy as before. The complete equilibrium pair distribution function is used to determine the rate of dissipation of energy due to inelastic collisions. The results are compared with hard sphere MD simulations, and also with earlier theoretical and simulation studies.

\section{ANALYSIS}

The system consists of a bed of circular disks (of diameter $\sigma$ ) in a gravitational field driven by a vibrating surface. The vibrating surface has a periodic amplitude function but no assumption is made regarding the form of the function. There is a source of energy at the vibrating surface due to particle collisions with the surface, and the dissipation is due to inelastic collisions. A balance between the two determines the "temperature", which is the mean square velocity of the particles.

The limit of low dissipation, where the coefficient of restitution $e$ is close to 1 , is considered. In this limit, it can be shown that the mean square velocity of the particles is large compared to the mean square velocity of the vibrating surface. An asymptotic expansion in the parameter $\epsilon \equiv U_{0}^{2} / T_{0}$ is used [8]. If the source and dissipation of energy are neglected in the leading approximation, the system resembles a gas of hard disks at equilibrium in a gravitational field. The velocity distribution function is a Maxwell-Boltzmann distribution at equilibrium

$$
F(\mathbf{u})=\frac{1}{2 \pi T_{0}} \exp \left(-\frac{u^{2}}{2 T_{0}}\right)
$$

where $T_{0}$ is the leading order temperature. The density profile is determined by solving the momentum balance equation in the vertical direction

$$
\frac{\partial p}{\partial z}-\rho g=0
$$

where $p$ is the pressure, $\rho$ is the density (number of particles per area) and $g$ is the acceleration due to gravity. For a gas at equilibrium, the pressure is related to the density by the virial equation of state, which in the case of inelastic circular disks is

$$
p=\rho T_{0}\left[\frac{1+e}{2}+(1+e) g_{0}(\nu) \nu\right],
$$

where $g_{0}(\nu)$ is the pair distribution function at contact, which for circular disks is given by [13]

$$
g_{0}(\nu)=\frac{1}{16(1-\nu)^{2}}\left[16-7 \nu-\frac{\nu^{3}}{4(1-\nu)^{2}}\right]
$$

and $\nu$ is the area fraction corresponding to $\rho$. If the coefficient of restitution is set equal to 1 in the leading approximation, the equation for the pressure reduces to the standard virial equation of state

$$
p=\rho T_{0}\left[1+2 g_{0}(\nu) \nu\right] .
$$

The resulting equation from Eq. (2) for the density profile is a first order ordinary differential equation, which can be solved using the mass conservation condition

$$
\int_{0}^{\infty} d z \rho=N
$$

where $N$ is the number of particles per unit width of the bed. Note that the leading order temperature $T_{0}$ is still unknown at this stage. This is determined using a balance between the source and dissipation of energy. The source of energy due to particle collisions with the vibrating surface is determined using an equilibrium average over the increase in energy due to particle collisions with the vibrating surface [8, 10]

$$
S_{0}=\left.2 \sqrt{\frac{2}{\pi}} T_{0}^{1 / 2}\left\langle U^{2}\right\rangle g_{0}(\nu) \rho\right|_{z=0} .
$$

Here $\left\langle U^{2}\right\rangle$ represents the mean square velocity of the vibrating surface. The rate of dissipation of energy per unit width is calculated by averaging over the energy loss over all the collisions between particles and integrating over the height of the bed [8]

$$
D_{0}=\sqrt{\pi} \sigma\left(1-e^{2}\right) T_{0}^{3 / 2} \int_{0}^{\infty} d z g_{0}(\nu) \rho^{2} .
$$

Note that the $g_{0}$ appearing in $S_{0}$ and $D_{0}$ is the Enskog factor which accounts for the increase in the frequency of collision for hard disks at high densities. The temperature $T_{0}$ can now be determined from the relation

$$
S_{0}=D_{0}
$$

An analytical solution to the density variation Eq. (2) can be determined in the low density limit using the equation of state for an ideal gas for the pressure [8].

$$
\rho=\frac{N g}{T_{0}} \exp \left(-\frac{g z}{T_{0}}\right)
$$

where the leading order temperature is given by,

$$
T_{0}=\frac{4 \sqrt{2}}{\pi} \frac{\left\langle U^{2}\right\rangle}{N \sigma\left(1-e^{2}\right)} .
$$

In the low-density limit the density decays exponentially from the bottom of the bed. At higher densities the solution to the density variation is no longer exponential throughout, and has to be obtained numerically by an iterative scheme. However, at large distances from the bottom, the bed is dilute and the 
ideal gas law holds good, hence the decay is exponential, even though near the bottom it is not. This gives a convenient starting point for the numerical integration from a finite height, above which we assume the asymptotic solution $(z \rightarrow \infty)$ to be given by an exponential decay known to within two undetermined constants. A value for the density and the temperature is assumed at this height and the integration is carried out up to the vibrating plate $(z=0)$. The complete density profile is obtained by combining the numerical and the asymptotic solutions. If the conditions Eq. (6) and Eq. (9) are not satisfied after one such integration, a new value is determined for the density and temperature using the Newton-Raphson method, and the iteration is repeated till convergence. In cases where the convergence is poor, the solution is obtained by continuing a low density solution in a parameter such as $N \sigma$ or $U_{0}$.

Viscous dissipation: The above analysis can be easily extended to the case of dissipation purely due to viscous drag. The expression for the source of energy remains the same as given by Eq. (7). A drag law given by $a_{i}=-\mu u_{i}$ is assumed. The total leading order rate of dissipation per unit width will then be

$$
\begin{aligned}
D_{D 0} & =\mu \int_{0}^{\infty} d z \rho \int d \mathbf{u} F(\mathbf{u}) \mathbf{u} \cdot \mathbf{u} \\
& =2 \mu N T_{0}
\end{aligned}
$$

Unlike Eq. (8), the leading order dissipation is the same for the low density and the high density cases. Nevertheless, the density profile has to be obtained numerically in the manner outlined above, with Eq. (12) substituted for Eq. (8) in Eq. (9).

\section{SIMULATION AND RESULTS}

The hard sphere molecular dynamics (MD), also known as event driven (ED) method [9] is used for the simulations of the vibro-fluidised bed. Periodic boundary conditions are used in the horizontal direction and the vibrating surface at the bottom has a sawtooth form for the amplitude function. The simulations are carried out only for the case of inelastic collisions, since the viscous drag requires a different treatment than the ED method.

The density profiles obtained using the present analysis, as well as the earlier low density approximations of Kumaran [8], are compared with the simulation results in Figs. 11 and 2. It is seen that the density profiles of the present analysis are in good agreement with the simulation results even when the density near the bottom of the bed becomes large, while the profiles from the low density approximation have significant errors. Fig. 3 shows the nature of the density profile in the high density limit in the case of dissipation due to viscous drag. Here too the present analysis gives reasonable values for packing fraction near the bottom, while the low density theory predicts physically incorrect values.

In a recent work, McNamara and Luding [11] reported the scaling of dissipation with the center of mass obtained from simulations. The results agreed with the low density theory of

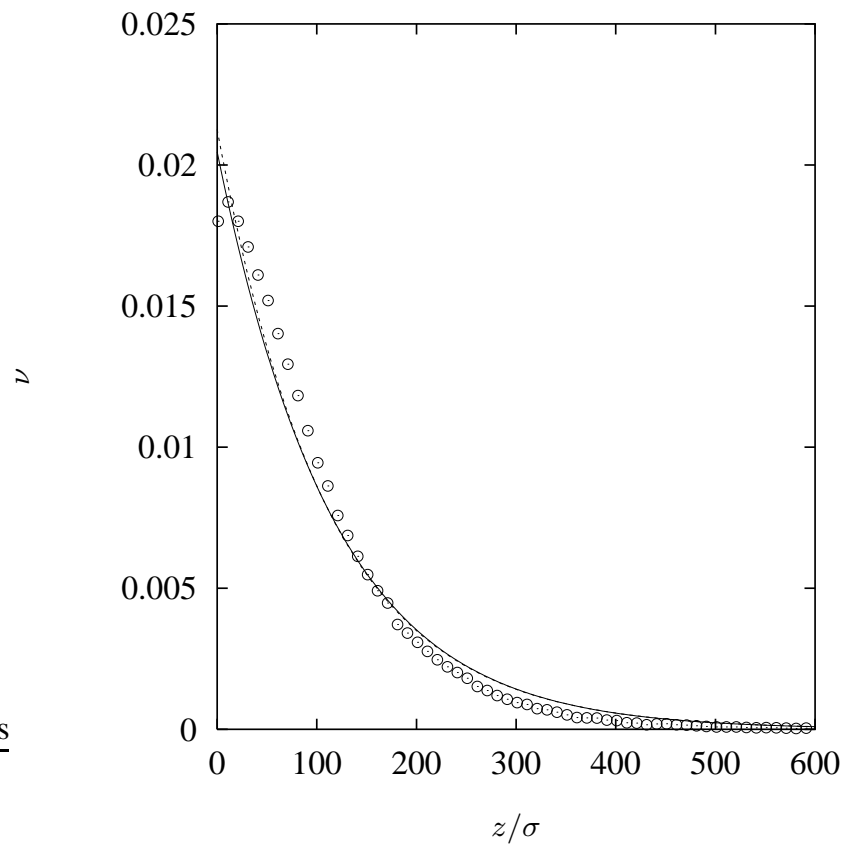

FIG. 1. Exponential decay of packing fraction $(\nu)$ with a normalised height $(z / \sigma)$ at low densities. The predictions of the present analysis (solid line) and the low density theory (dotted line) of [8] is compared with simulation (points). Both the predictions are nearly identical. Here, $\epsilon=0.3, N \sigma=3, g=1$, and $U_{0}=6$.

[10] but a systematic deviation was observed at high densities in all the cases. This deviation is captured in the present analysis. The leading order dissipation at low densities in the bed is given by [8]

$$
D_{0}=\frac{\sqrt{\pi}}{2}\left(1-e^{2}\right) N^{2} \sigma g \sqrt{T_{0}} .
$$

In [11] the total dissipation obtained from the simulation was normalised by a factor taken out from this leading order dissipation and a non dimensional number was defined as

$$
C_{p p} \equiv \frac{D_{0}}{(1-e) N^{2} \sigma g \sqrt{T_{0} / 2}}
$$

The scaling of this factor with the height of the center of mass (h) above the position at rest $\left(h_{0}\right)$ was studied. This factor was found out for different parameter sets by varying the bottom wall velocity $U_{0}$ over several decades such that the bed is taken from a densely packed regime to a very low density regime. They chose a central data set and varied the parameters one at a time. It was found that in all the cases considered, the scaling relation collapsed to a single curve. The central parameter set has the following values $N=3.2, \sigma=1, g=1$, $e=0.95$.

The present analysis is valid when $\epsilon \equiv U_{0}^{2} / T_{0} \ll 1$ and when the frequency of particle-particle collision is much greater than the frequency of particle-wall collisions. It can 


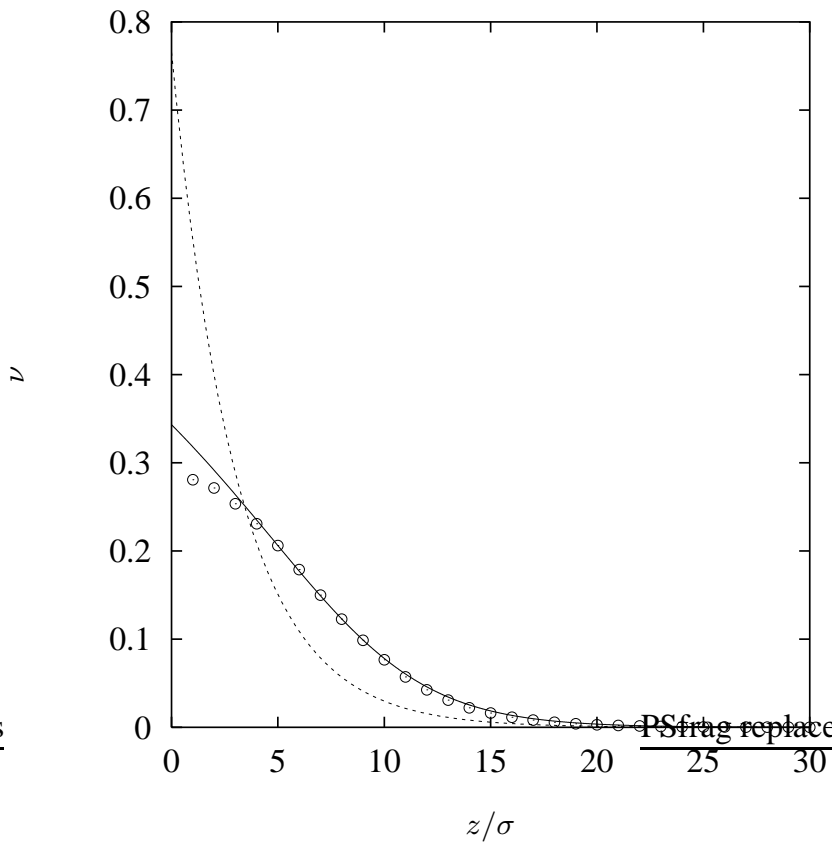

FIG. 2. Deviation of the density profile from the exponential decay at high densities in the case of dissipation due to inelastic collisions. The simulation result (points) is captured by the present analysis (solid line) which is lower than the exponential decay (dotted line) of the low density theory of [8] near the bottom of the bed. Here $\epsilon=0.3, N \sigma=3, g=1$, and $U_{0}=1$.

be shown that in the leading order the ratio of the frequency of particle-particle collisions to the frequency particle-wall collisions is $\sqrt{2} \pi N \sigma$. Hence the present analysis will hold good when $N \sigma \gg 1 / \sqrt{2} \pi$. The central set corresponds to $\epsilon=0.35, N \sigma=3.2$ and therefore we expect the present analysis to hold good for this case. Most of the parameter sets used in [1]] also fall within the limits of the theory derived here.

Fig. 4 shows the theoretical predictions of the total dissipation for the different cases reported in Fig. 2 in [11]. It is compared with the results of two simulations in Fig. 5 . It is seen that the present analysis correctly predicts the lowering of the coefficient $C_{p p}$ at high densities. This reduction in the dissipation from the constant value at low densities is the net result of two opposing factors: (i) decrease in the density from the exponential behaviour near the vibrating bottom (see Fig. 2), hence reducing the total value of the dissipation, and (ii) increase in frequency of collisions at high densities, increasing the dissipation.

It is also seen that not all the theoretical predictions collapse on to a curve as is the case with the data from the simulation. In two of the cases the theory does not agree with the simulations because (i) in one the value of the perturbation parameter is high $(\epsilon=1.73)$ and the leading order theory is valid only for low $\epsilon$, and (ii) in the other case the value of $N \sigma=0.65$ is low.

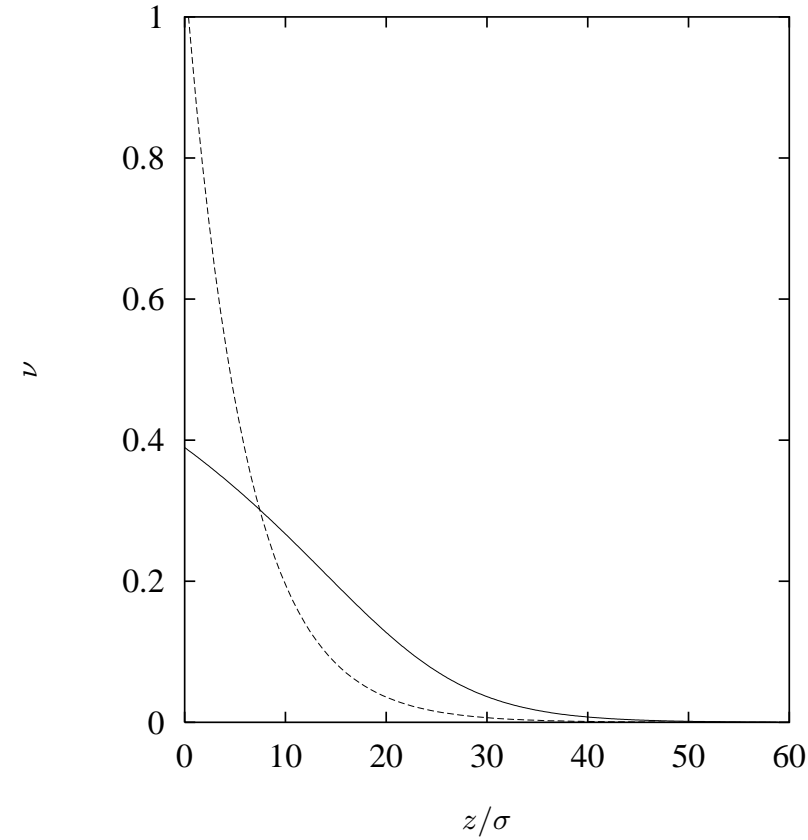

FIG. 3. Deviation of the density profile from the exponential decay at high densities in the case of dissipation due to viscous drag. The present analysis (solid line) gives physically plausible values for the packing fraction near the bottom, while the low density theory (dotted line) of [8] predicts values higher than the maximum closed packing. Here $\epsilon=0.2, N \sigma=20, g=20, \mu=0.1$, and $U_{0}=5$.

In Fig. 月, the apparent mismatch with 'e-' is not a discrepancy with the model, but has got to do with the formula chosen used in [11] for the normalisation of the dissipation factor $C_{p p}$. They had chosen to normalise the dissipation by a factor $(1-e)$. While this might have given a better fit for high densities (low center of mass), the correct factor for very low densities is $\left(1-e^{2}\right)$ as given by Eq. (13). The difference is more pronounced in the case of $e \ll 1$, which, here, has a value $e=0.75$. A close inspection of the curves 'e-' in Fig. $\exists$ and Fig. 5 show that the theory and simulation do indeed agree with each other.

We also note here that the data taken from the reported simulation [1]] is for asymmetric sawtooth vibration, whereas our simulation is for the symmetric sawtooth. Both these give similar results for the scaling of $C_{p p}$. Also the theoretical predictions for the symmetric and the asymmetric sawtooth are identical, indicating that the form of the bottom wall vibration does not affect the scaling of the dissipation with the center of mass.

\section{CONCLUSION}

In summary, a theory to describe the state of a vibrofluidised bed in the dense limit was derived. This is different from the earlier theory of Kumaran [8, 10], which is valid for 


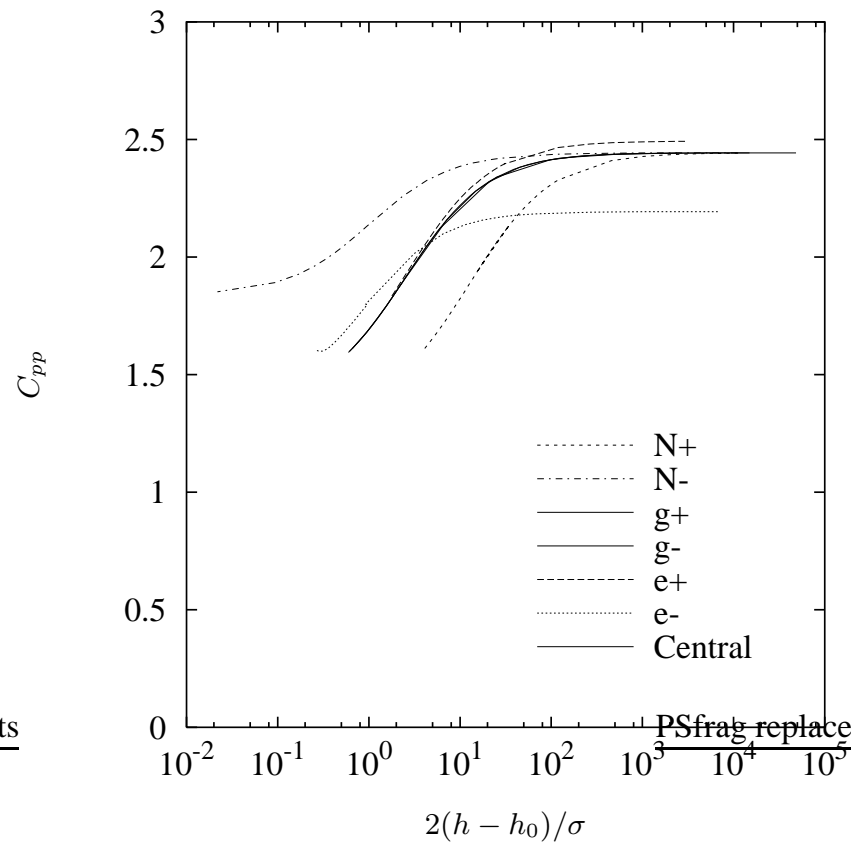

FIG. 4. Theoretical scaling of the normalised dissipation $\left(C_{p p}\right)$ against the center of mass $(h)$ above the position at rest $\left(h_{0}\right)$ for the different cases reported in [11]. All except two- $(\mathrm{N}+)$ with $\epsilon=1.73$ and $(\mathrm{N}-)$ with $N \sigma=0.65$ collapse on to a single curve in the linear region. The parameters indicated correspond to $N=16(\mathrm{~N}+)$, $N=0.65(\mathrm{~N}-), g=25(\mathrm{~g}+), g=0.04(\mathrm{~g}-), e=0.99(\mathrm{e}+)$, $e=0.75(\mathrm{e}-)$, rest of the parameters being same as the one in the central set, which has the following values $N=3.2, \sigma=1, g=1$, $e=0.95$.

low densities where the ideal gas equation was used and the pair distribution function was set equal to 1 . We have made use of the virial equation of state to obtain a correction to the exponential density profile obtained in low densities and the pair distribution function is used to calculate the increased frequency of collisions in the source and the dissipation of energy. The theoretical predictions of density and temperature were compared with the results obtained from MD simulation of two dimensional disks. The theory correctly predicts the lowering of the density from the exponential value at high densities near the bottom. The theory also predicts the scaling relations of the total dissipation in the bed reported in [11].

[1] H. M. Jaeger and S. R. Nagel, Rev. Mod. Phys. 68, 1259 (1996).

[2] S. Warr, J. M. Huntley, and G. T. H. Jackques, Phys. Rev. E 52, 5583 (1995).

[3] F. Melo, P. B. Umbanhowar, and H. L. Swinney, Phys. Rev. E 75, 3838 (1995).

[4] T. Shrinbot, Nature 383, 574 (1997).

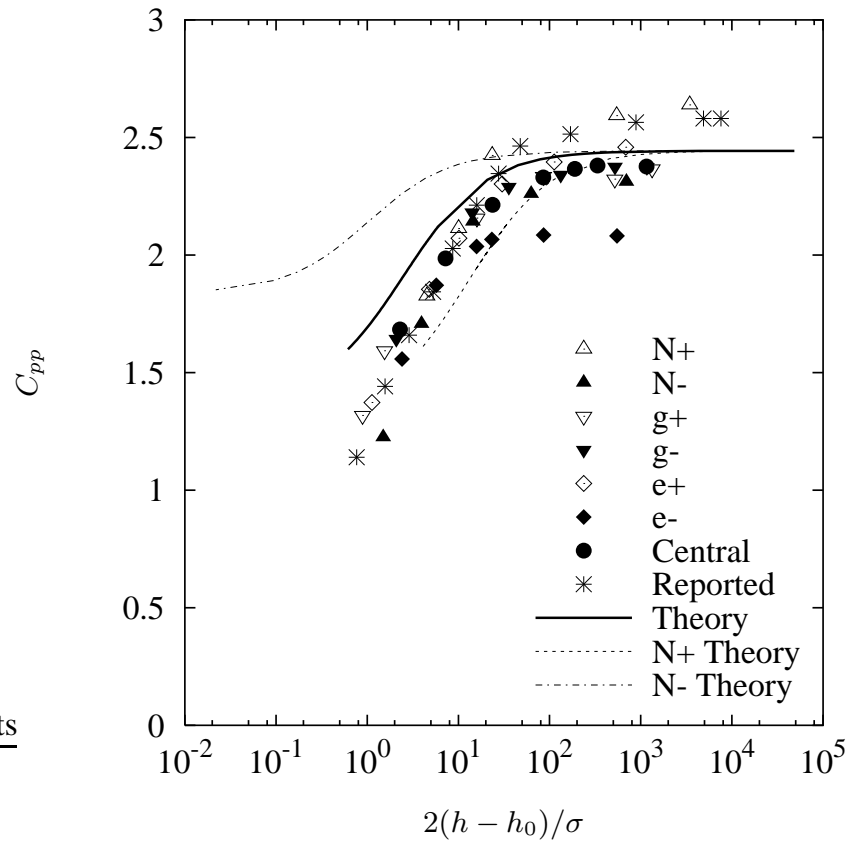

FIG. 5. Scaling of the normalised dissipation with the center of mass: Predictions from the present analysis is compared with the results from our simulations and the reported results in [11]. The linear portion of all the curves from theory, except two, fall on the solid line denoted as 'Theory'. The two exceptions are also shown. A set of points correspond to the simulation data with parameter values $N=16(\mathrm{~N}+), N=0.65(\mathrm{~N}-), g=25(\mathrm{~g}+), g=0.04(\mathrm{~g}-)$, $e=0.99(\mathrm{e}+), e=0.75(\mathrm{e}-)$; rest of the parameters in a set being the same as the one in the central set, which has the following values $N=3.2, \sigma=1, g=1, e=0.95$.

[5] S. Tsimring and I. S. Aranson, Phys. Rev. Lett. 79, 213 (1997).

[6] S. C. Venkataramani and E. Ott, Phys. Rev. Lett. 80, 3495 (1998).

[7] J. T. Jenkins and S. B. Savage, J. Fluid Mech. 130, 187 (1983).

[8] V. Kumaran, J. Fluid Mech. 364, 163 (1998).

[9] S. Luding, H. J. Herrmann, and A. Blumen, Phys. Rev. E 50, 3100 (1994).

[10] V. Kumaran, Phys. Rev. E 57, 5660 (1998).

[11] S. McNamara and S. Luding, Phys. Rev. E 58, 813 (1998).

[12] J. M. Huntley, Phys. Rev. E 58, 5168 (1998).

[13] L. Verlet and D. Levesque, Mol. Phys. 46, 969 (1982). 